\title{
The maximum modulus of a trigonometric trinomial
}

\author{
Stefan Neuwirth
}

\begin{abstract}
Let $\Lambda$ be a set of three integers and let $\mathscr{C}_{\Lambda}$ be the space of $2 \pi$-periodic functions with spectrum in $\Lambda$ endowed with the maximum modulus norm. We isolate the maximum modulus points $x$ of trigonometric trinomials $T \in \mathscr{C}_{\Lambda}$ and prove that $x$ is unique unless $|T|$ has an axis of symmetry. This permits to compute the exposed and the extreme points of the unit ball of $\mathscr{C}_{\Lambda}$, to describe how the maximum modulus of $T$ varies with respect to the arguments of its Fourier coefficients and to compute the norm of unimodular relative Fourier multipliers on $\mathscr{C}_{\Lambda}$. We obtain in particular the Sidon constant of $\Lambda$.
\end{abstract}

\section{Introduction}

Let $\lambda_{1}, \lambda_{2}$ and $\lambda_{3}$ be three pairwise distinct integers. Let $r_{1}, r_{2}$ and $r_{3}$ be three positive real numbers. Given three real numbers $t_{1}, t_{2}$ and $t_{3}$, let us consider the trigonometric trinomial

$$
T(x)=r_{1} \mathrm{e}^{\mathrm{i}\left(t_{1}+\lambda_{1} x\right)}+r_{2} \mathrm{e}^{\mathrm{i}\left(t_{2}+\lambda_{2} x\right)}+r_{3} \mathrm{e}^{\mathrm{i}\left(t_{3}+\lambda_{3} x\right)}
$$

for $x \in \mathbb{R}$. The $\lambda$ 's are the frequencies of the trigonometric trinomial $T$, the $r$ 's are the moduli or intensities and the t's the arguments or phases of its Fourier coefficients $r_{1} \mathrm{e}^{\mathrm{i} t_{1}}, r_{2} \mathrm{e}^{\mathrm{i} t_{2}}$ and $r_{3} \mathrm{e}^{\mathrm{i} t_{3}}$.

The maximum modulus of a trigonometric trinomial has a geometric interpretation. Without loss of generality, we may assume that $\lambda_{2}$ is between $\lambda_{1}$ and $\lambda_{3}$. Let $H$ be the curve with complex equation

$$
z=r_{1} \mathrm{e}^{\mathrm{i}\left(t_{1}-\left(\lambda_{2}-\lambda_{1}\right) x\right)}+r_{3} \mathrm{e}^{\mathrm{i}\left(t_{3}+\left(\lambda_{3}-\lambda_{2}\right) x\right)} \quad(-\pi<x \leqslant \pi) .
$$

$H$ is a hypotrochoid: it is drawn by a point at distance $r_{3}$ to the centre of a circle with radius $r_{1}\left|\lambda_{2}-\lambda_{1}\right| /\left|\lambda_{3}-\lambda_{2}\right|$ that rolls inside another circle with radius $r_{1}\left|\lambda_{3}-\lambda_{1}\right| /\left|\lambda_{3}-\lambda_{2}\right|$. The maximum modulus of (1) is the maximum distance of points $z \in H$ to a given point $-r_{2} \mathrm{e}^{\mathrm{i} t_{2}}$ of the complex plane. Figure 1 illustrates a particular case.

We deduce an interval on which $T$ attains its maximum modulus independently of the moduli of its Fourier coefficients (see Theorem 7.1 (a) for a detailed answer.) We prove in particular the following result. 


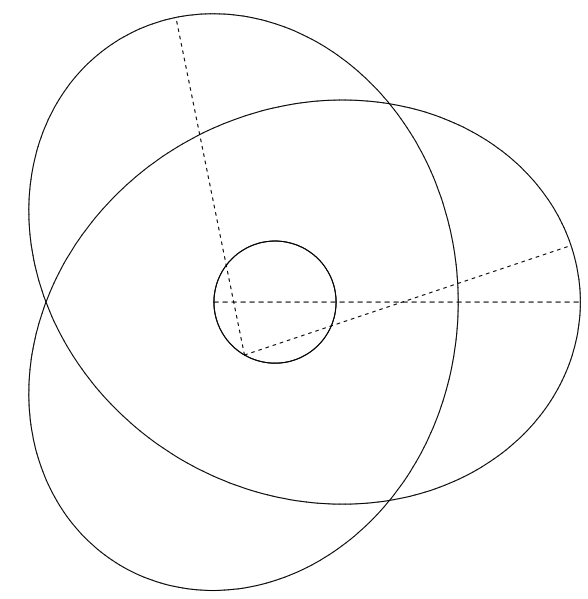

Figure 1: The unit circle, the hypotrochoid $z=4 \mathrm{e}^{-\mathrm{i} 2 x}+\mathrm{e}^{\mathrm{i} x}$, its unique point at maximum distance to -1 and its two points at maximum distance to $-\mathrm{e}^{\mathrm{i} \pi / 3}$.

Theorem 1.1. Let $d=\operatorname{gcd}\left(\lambda_{2}-\lambda_{1}, \lambda_{3}-\lambda_{2}\right)$ and let $\tau$ be the distance of

$$
\frac{\lambda_{2}-\lambda_{3}}{d} t_{1}+\frac{\lambda_{3}-\lambda_{1}}{d} t_{2}+\frac{\lambda_{1}-\lambda_{2}}{d} t_{3}
$$

to $2 \pi \mathbb{Z}$. The trigonometric trinomial $T$ attains its maximum modulus at a unique point modulo $2 \pi / d$, with multiplicity 2 , unless $\tau=\pi$.

Theorem 1.1 shows that if there are two points of the hypotrochoid $H$ at maximum distance to $-r_{2} \mathrm{e}^{\mathrm{i} t_{2}}$, it is so only because $-r_{2} \mathrm{e}^{\mathrm{i} t_{2}}$ lies on an axis of symmetry of $H$.

We obtain a precise description of those trigonometric trinomials that attain their maximum modulus twice modulo $2 \pi / d$ (see Theorem $7.1(c)$.) Their rôle is illustrated by the following geometric result. Let us first put up the proper functional analytic framework. Let $\Lambda=\left\{\lambda_{1}, \lambda_{2}, \lambda_{3}\right\}$ be the spectrum of the trigonometric trinomial $T$ and denote $\mathrm{e}_{\lambda}: x \mapsto \mathrm{e}^{\mathrm{i} \lambda x}$. Let $\mathscr{C}_{\Lambda}$ be the space of functions spanned by the $\mathrm{e}_{\lambda}$ with $\lambda \in \Lambda$, endowed with the maximum modulus norm. Recall that a point $P$ of a compact convex set $K$ is exposed if there is a hyperplane that meets $K$ only in $P$; $P$ is extreme if it is not the midpoint of any two other points of $K$.

Theorem 1.2. Let $K$ be the unit ball of the space $\mathscr{C}_{\Lambda}$ and let $P \in K$.

(a) The point $P$ is an exposed point of $K$ if and only if $P$ is either a trigonometric monomial $\mathrm{e}^{\mathrm{i} \alpha} \mathrm{e}_{\lambda}$ with $\alpha \in \mathbb{R}$ and $\lambda \in \Lambda$ or a trigonometric trinomial that attains its maximum modulus, 1 , at two points modulo $2 \pi / d$. Every linear functional on $\mathscr{C}_{\Lambda}$ attains its norm on an exposed point of $K$.

(b) The point $P$ is an extreme point of $K$ if and only if $P$ is either a trigonometric monomial $\mathrm{e}^{\mathrm{i} \alpha} \mathrm{e}_{\lambda}$ with $\alpha \in \mathbb{R}$ and $\lambda \in \Lambda$ or a trigonometric trino- 
mial such that $1-|P|^{2}$ has four zeroes modulo $2 \pi / d$, counted with multiplicities.

We describe the dependence of the maximum modulus of the trigonometric trinomial $T$ on the arguments. The general issue has been addressed for a long time. The articles [10, 17] are two early references.

Let us state our main theorem, that solves an elementary case of the complex Mandel'shtam problem, a term coined in [5. It appeared originally in electrical circuit theory: "L. I. Mandel'shtam communicated me a problem on the phase choice of electric currents with different frequencies such that the capacity of the resulting current to blow is minimal" [4, p. 396].

Extremal problem 1.3 (Complex Mandel'shtam problem). To find the minimum of the maximum modulus of a trigonometric polynomial with given Fourier coefficient moduli.

Theorem 1.4. The maximum modulus of (1) is a strictly decreasing function of $\tau$. In particular,

$$
\begin{aligned}
\min _{t_{1}, t_{2}, t_{3}} \max _{x}\left|r_{1} \mathrm{e}^{\mathrm{i}\left(t_{1}+\lambda_{1} x\right)}+r_{2} \mathrm{e}^{\mathrm{i}\left(t_{2}+\lambda_{2} x\right)}+r_{3} \mathrm{e}^{\mathrm{i}\left(t_{3}+\lambda_{3} x\right)}\right| \\
\quad=\max _{x}\left|\epsilon_{1} r_{1} \mathrm{e}^{\mathrm{i} \lambda_{1} x}+\epsilon_{2} r_{2} \mathrm{e}^{\mathrm{i} \lambda_{2} x}+\epsilon_{3} r_{3} \mathrm{e}^{\mathrm{i} \lambda_{3} x}\right|
\end{aligned}
$$

if $\epsilon_{1}, \epsilon_{2}$ and $\epsilon_{3}$ are real signs +1 or -1 such that $\epsilon_{i} \epsilon_{j}=-1$, where $i, j, k$ is a permutation of $1,2,3$ such that the power of 2 in $\lambda_{i}-\lambda_{j}$ is greater than the power of 2 in $\lambda_{i}-\lambda_{k}$ and in $\lambda_{k}-\lambda_{j}$.

Our result shows that the maximum modulus is minimal when the phases are chosen in opposition, independently of the intensities $r_{1}, r_{2}$ and $r_{3}$.

The decrease of the maximum modulus of (11) may be bounded as shown in the next result.

Theorem 1.5. Suppose that $\lambda_{2}$ is between $\lambda_{1}$ and $\lambda_{3}$. The quotient of the maximum modulus of (11) by $\left|r_{1}+r_{2} \mathrm{e}^{\mathrm{i} \tau d /\left|\lambda_{3}-\lambda_{1}\right|}+r_{3}\right|$ is a strictly increasing function of $\tau$ unless $r_{1}: r_{3}=\left|\lambda_{3}-\lambda_{2}\right|:\left|\lambda_{2}-\lambda_{1}\right|$, in which case it is constantly equal to 1.

When $r_{1}: r_{3}=\left|\lambda_{3}-\lambda_{2}\right|:\left|\lambda_{2}-\lambda_{1}\right|$, the hypotrochoid $H$ with equation (2) is a hypocycloid with $\left|\lambda_{3}-\lambda_{1}\right| / d$ cusps: the rolling point is on the rolling circle. Figure 2 illustrates a particular case.

We may deduce from Theorem 1.5 a less precise but handier inequality.

Theorem 1.6. Let

$$
D=\frac{\max \left(\left|\lambda_{2}-\lambda_{1}\right|,\left|\lambda_{3}-\lambda_{2}\right|,\left|\lambda_{3}-\lambda_{1}\right|\right)}{\operatorname{gcd}\left(\lambda_{2}-\lambda_{1}, \lambda_{3}-\lambda_{1}\right)}
$$




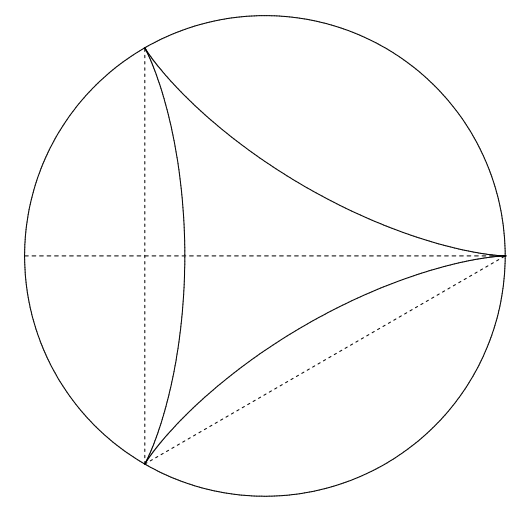

Figure 2: The unit circle, the deltoid $z=(1 / 3) \mathrm{e}^{-\mathrm{i} 2 x}+(2 / 3) \mathrm{e}^{\mathrm{i} x}$, its unique point at maximum distance to -1 and its two points at maximum distance to $-\mathrm{e}^{\mathrm{i} \pi / 3}$.

be the quotient of the diameter of $\Lambda$ by $d$. Let $t_{1}^{\prime}, t_{2}^{\prime}$ and $t_{3}^{\prime}$ be another three real numbers and define similarly $\tau^{\prime}$. If $\tau>\tau^{\prime}$, then

$$
\begin{aligned}
\max _{x}\left|r_{1} \mathrm{e}^{\mathrm{i}\left(t_{1}+\lambda_{1} x\right)}+r_{2} \mathrm{e}^{\mathrm{i}\left(t_{2}+\lambda_{2} x\right)}+r_{3} \mathrm{e}^{\mathrm{i}\left(t_{3}+\lambda_{3} x\right)}\right| \\
\quad \frac{\cos (\tau / 2 D)}{\cos \left(\tau^{\prime} / 2 D\right)} \max _{x}\left|r_{1} \mathrm{e}^{\mathrm{i}\left(t_{1}^{\prime}+\lambda_{1} x\right)}+r_{2} \mathrm{e}^{\mathrm{i}\left(t_{2}^{\prime}+\lambda_{2} x\right)}+r_{3} \mathrm{e}^{\mathrm{i}\left(t_{3}^{\prime}+\lambda_{3} x\right)}\right|
\end{aligned}
$$

with equality if and only if $r_{1}: r_{2}: r_{3}=\left|\lambda_{3}-\lambda_{2}\right|:\left|\lambda_{3}-\lambda_{1}\right|:\left|\lambda_{2}-\lambda_{1}\right|$.

If we choose $\tau^{\prime}=0$ in the last result, we get the solution to an elementary case of the following extremal problem.

Extremal problem 1.7. To find the minimum of the maximum modulus of a trigonometric polynomial with given spectrum, Fourier coefficient arguments and moduli sum.

Theorem 1.8. We have

$$
\frac{\max _{x}\left|r_{1} \mathrm{e}^{\mathrm{i}\left(t_{1}+\lambda_{1} x\right)}+r_{2} \mathrm{e}^{\mathrm{i}\left(t_{2}+\lambda_{2} x\right)}+r_{3} \mathrm{e}^{\mathrm{i}\left(t_{3}+\lambda_{3} x\right)}\right|}{r_{1}+r_{2}+r_{3}} \geqslant \cos (\tau / 2 D)
$$

with equality if and only if $\tau=0$ or $r_{1}: r_{2}: r_{3}=\left|\lambda_{3}-\lambda_{2}\right|:\left|\lambda_{3}-\lambda_{1}\right|:\left|\lambda_{2}-\lambda_{1}\right|$.

The dependence of the maximum modulus of (1) on the arguments may also be expressed as properties of relative multipliers. Given three real numbers $t_{1}$, $t_{2}$ and $t_{3}$, the linear operator on $\mathscr{C}_{\Lambda}$ defined by $\mathrm{e}_{\lambda_{j}} \mapsto \mathrm{e}^{\mathrm{i} t_{j}} \mathrm{e}_{\lambda_{j}}$ is a unimodular relative Fourier multiplier: it multiplies each Fourier coefficient of elements of $\mathscr{C}_{\Lambda}$ by a fixed unimodular number; let us denote it by $\left(t_{1}, t_{2}, t_{3}\right)$. Consult 7 for general background on relative multipliers.

Theorem 1.9. The unimodular relative Fourier multiplier $\left(t_{1}, t_{2}, t_{3}\right)$ has norm

$$
\cos ((\pi-\tau) / 2 D) / \cos (\pi / 2 D),
$$


where $\tau$ is defined as in Theorem 1.1 and $D$ is given by (4), and attains its norm exactly at functions of the form

$$
\begin{gathered}
r_{1} \mathrm{e}^{\mathrm{i}\left(u_{1}+\lambda_{1} x\right)}+r_{2} \mathrm{e}^{\mathrm{i}\left(u_{2}+\lambda_{2} x\right)}+r_{3} \mathrm{e}^{\mathrm{i}\left(u_{3}+\lambda_{3} x\right)} \\
\text { with } r_{1}: r_{2}: r_{3}=\left|\lambda_{3}-\lambda_{2}\right|:\left|\lambda_{3}-\lambda_{1}\right|:\left|\lambda_{2}-\lambda_{1}\right| \text { and } \\
\frac{\lambda_{2}-\lambda_{3}}{d} u_{1}+\frac{\lambda_{3}-\lambda_{1}}{d} u_{2}+\frac{\lambda_{1}-\lambda_{2}}{d} u_{3}=\pi \quad \bmod 2 \pi .
\end{gathered}
$$

The maximum of the norm of unimodular relative Fourier multipliers is the complex unconditional constant of the canonical basis $\left(\mathrm{e}_{\lambda_{1}}, \mathrm{e}_{\lambda_{2}}, \mathrm{e}_{\lambda_{3}}\right)$ of $\mathscr{C}_{\Lambda}$. As

$$
r_{1}+r_{2}+r_{3}=\max _{x}\left|r_{1} \mathrm{e}^{\mathrm{i} \lambda_{1} x}+r_{2} \mathrm{e}^{\mathrm{i} \lambda_{2} x}+r_{3} \mathrm{e}^{\mathrm{i} \lambda_{3} x}\right|,
$$

this constant is the minimal constant $C$ such that

$$
r_{1}+r_{2}+r_{3} \leqslant C \max _{x}\left|r_{1} \mathrm{e}^{\mathrm{i}\left(u_{1}+\lambda_{1} x\right)}+r_{2} \mathrm{e}^{\mathrm{i}\left(u_{2}+\lambda_{2} x\right)}+r_{3} \mathrm{e}^{\mathrm{i}\left(u_{3}+\lambda_{3} x\right)}\right| ;
$$

it is therefore the Sidon constant of $\Lambda$. It is also the solution to the following extremal problem.

Extremal problem 1.10 (Sidon constant problem). To find the minimum of the maximum modulus of a trigonometric polynomial with given spectrum and Fourier coefficient moduli sum.

We obtain the following result, setting $\tau=\pi$ in Theorem 1.9.

Corollary 1.11. The Sidon constant of $\Lambda$ is $\sec (\pi d / 2 D)$. It is attained exactly at functions of the form given in Theorem 1.9.

Let us now give a brief description of this article. In Sections 2 and 3, we use carefully the invariance of the maximum modulus under rotation, translation and conjugation to reduce the arguments $t_{1}, t_{2}$ and $t_{3}$ of the Fourier coefficients of the trigonometric trinomial $T$ to the variable $\tau$. Section 4 shows how to further reduce this study to the trigonometric trinomial

$$
r_{1} \mathrm{e}^{-\mathrm{i} k x}+r_{2} \mathrm{e}^{\mathrm{i} t}+r_{3} \mathrm{e}^{\mathrm{i} l x}
$$

with $k$ and $l$ positive coprime integers and $t \in[0, \pi /(k+l)]$. In Section 5 we prove that (5) attains its maximum modulus for $x \in[-t / k, t / l]$. Section [6] studies the variations of the modulus of (5) for $x \in[-t / k, t / l]$ : it turns out that it attains its absolute maximum only once on that interval. This yields Theorem 1.1. Section 7 restates the results of the two previous sections for a general trigonometric trinomial $T$. Section 8 is dedicated to the proof of Theorem 1.2 In Section 9, we compute the directional derivative of the maximum modulus of (5) with respect to the argument $t$ and prove Theorems 1.4, 1.5, 1.6 and 1.8. In Section 10, we prove Theorem 1.9 and show how to lift unimodular relative Fourier multipliers to operators of convolution with a linear combination of two Dirac measures. Section 11 replaces our computation of the Sidon constant in a general context; it describes the initial motivation for this research.

Part of these results appeared previously, with a different proof, in 14, Chapter II.10] and in [12]. 
Notation. Throughout this article, $\lambda_{1}, \lambda_{2}$ and $\lambda_{3}$ are three pairwise distinct integers, $\Lambda=\left\{\lambda_{1}, \lambda_{2}, \lambda_{3}\right\}$ and $d=\operatorname{gcd}\left(\lambda_{2}-\lambda_{1}, \lambda_{3}-\lambda_{2}\right)$. If $\lambda$ is an integer, $\mathrm{e}_{\lambda}$ is the function $x \mapsto \mathrm{e}^{\mathrm{i} \lambda x}$ of the real variable $x$. A trigonometric polynomial is a linear combination of functions $\mathrm{e}_{\lambda}$; it is a monomial, binomial or trinomial if this linear combination has one, two or three nonzero coefficients, respectively. The normed space $\mathscr{C}_{\Lambda}$ is the three-dimensional space of complex functions spanned by $\mathrm{e}_{\lambda}$ with $\lambda \in \Lambda$, endowed with the maximum modulus norm. The Dirac measure $\delta_{x}$ is the linear functional $T \mapsto T(x)$ of evaluation at $x$ on the space of continuous functions.

\section{Isometric relative Fourier multipliers}

The rôle of Quantity (3) is explained by the following lemma.

Lemma 2.1. Let $t_{1}, t_{2}$ and $t_{3}$ be real numbers. The unimodular relative Fourier multiplier $M=\left(t_{1}, t_{2}, t_{3}\right)$ is an isometry on $\mathscr{C}_{\Lambda}$ if and only if

$$
\frac{\lambda_{2}-\lambda_{3}}{d} t_{1}+\frac{\lambda_{3}-\lambda_{1}}{d} t_{2}+\frac{\lambda_{1}-\lambda_{2}}{d} t_{3} \in 2 \pi \mathbb{Z}:
$$

it is a unimodular multiple of a translation: there are real numbers $\alpha$ and $v$ such that $M f(x)=\mathrm{e}^{\mathrm{i} \alpha} f(x-v)$ for all $f \in \mathscr{C}_{\Lambda}$ and all $x \in \mathbb{R}$.

Proof. If $M$ is a unimodular multiple of a translation by a real number $v$, then

$$
\left|r_{1} \mathrm{e}^{\mathrm{i}\left(t_{1}+\lambda_{1} v\right)}+r_{2} \mathrm{e}^{\mathrm{i}\left(t_{2}+\lambda_{2} v\right)}+r_{3} \mathrm{e}^{\mathrm{i}\left(t_{3}+\lambda_{3} v\right)}\right|=r_{1}+r_{2}+r_{3},
$$

which holds if and only if

$$
t_{1}+\lambda_{1} v=t_{2}+\lambda_{2} v=t_{3}+\lambda_{3} v \quad \text { modulo } 2 \pi .
$$

There is a $v$ satisfying (7) if and only if Equation (6) holds as (7) means that there exist integers $a_{1}$ and $a_{3}$ such that

$$
v=\frac{t_{2}-t_{1}+2 \pi a_{1}}{\lambda_{1}-\lambda_{2}}=\frac{t_{2}-t_{3}+2 \pi a_{3}}{\lambda_{3}-\lambda_{2}} .
$$

If $t_{1}, t_{2}$ and $t_{3}$ are three real numbers satisfying ([6), let $v$ be such that (7) holds. Then

$$
\begin{aligned}
& r_{1} \mathrm{e}^{\mathrm{i}\left(t_{1}+u_{1}+\lambda_{1} x\right)}+r_{2} \mathrm{e}^{\mathrm{i}\left(t_{2}+u_{2}+\lambda_{2} x\right)}+r_{3} \mathrm{e}^{\mathrm{i}\left(t_{3}+u_{3}+\lambda_{3} x\right)} \\
& \quad=\mathrm{e}^{\mathrm{i}\left(t_{2}+\lambda_{2} v\right)}\left(r_{1} \mathrm{e}^{\mathrm{i}\left(u_{1}+\lambda_{1}(x-v)\right)}+r_{2} \mathrm{e}^{\mathrm{i}\left(u_{2}+\lambda_{2}(x-v)\right)}+r_{3} \mathrm{e}^{\mathrm{i}\left(u_{3}+\lambda_{3}(x-v)\right)}\right)
\end{aligned}
$$

for all real numbers $u_{1}, u_{2}, u_{3}$ and $x$. 


\section{The arguments of the Fourier coefficients of a trigonometric trinomial}

A translation and a rotation permit to reduce the three arguments of the Fourier coefficients of a trigonometric trinomial to just one variable. Use of the involution $\overline{f(-x)}$ of $\mathscr{C}_{\Lambda}$ permits to restrain even further the domain of that variable.

Lemma 3.1. Let $t_{1}, t_{2}$ and $t_{3}$ be real numbers and let $\tilde{t}_{2}$ be the representant of

$$
\frac{\lambda_{2}-\lambda_{3}}{\lambda_{3}-\lambda_{1}} t_{1}+t_{2}+\frac{\lambda_{1}-\lambda_{2}}{\lambda_{3}-\lambda_{1}} t_{3}
$$

modulo $2 \pi /\left|\lambda_{3}-\lambda_{1}\right|$ in $\left[-\pi d /\left|\lambda_{3}-\lambda_{1}\right|, \pi d /\left|\lambda_{3}-\lambda_{1}\right|[\right.$.

(a) There are real numbers $\alpha$ and $v$ such that

$$
\begin{aligned}
r_{1} \mathrm{e}^{\mathrm{i}\left(t_{1}+\lambda_{1} x\right)} & +r_{2} \mathrm{e}^{\mathrm{i}\left(t_{2}+\lambda_{2} x\right)}+r_{3} \mathrm{e}^{\mathrm{i}\left(t_{3}+\lambda_{3} x\right)} \\
& =\mathrm{e}^{\mathrm{i} \alpha}\left(r_{1} \mathrm{e}^{\mathrm{i} \lambda_{1}(x-v)}+r_{2} \mathrm{e}^{\mathrm{i}\left(\tilde{t}_{2}+\lambda_{2}(x-v)\right)}+r_{3} \mathrm{e}^{\left.\mathrm{i} \lambda_{3}(x-v)\right)}\right)
\end{aligned}
$$

for all $x$.

(b) Let $t=\left|\tilde{t}_{2}\right|$ be the distance of (8) to $\left(2 \pi d /\left|\lambda_{3}-\lambda_{1}\right|\right) \mathbb{Z}$. There is a sign $\epsilon \in\{+1,-1\}$ such that

$$
\begin{aligned}
& \left|r_{1} \mathrm{e}^{\mathrm{i}\left(t_{1}+\lambda_{1} x\right)}+r_{2} \mathrm{e}^{\mathrm{i}\left(t_{2}+\lambda_{2} x\right)}+r_{3} \mathrm{e}^{\mathrm{i}\left(t_{3}+\lambda_{3} x\right)}\right| \\
& =\left|r_{1} \mathrm{e}^{\mathrm{i} \lambda_{1} \epsilon(x-v)}+r_{2} \mathrm{e}^{\mathrm{i}\left(t+\lambda_{2} \epsilon(x-v)\right)}+r_{3} \mathrm{e}^{\left.\mathrm{i} \lambda_{3} \epsilon(x-v)\right)}\right|
\end{aligned}
$$

for all $x$.

Proof. (a). The argument $\tilde{t}_{2}$ is chosen so that the relative multiplier $\left(t_{1}, t_{2}-\right.$ $\left.\tilde{t}_{2}, t_{3}\right)$ is an isometry.

(b). If $\tilde{t}_{2}$ is negative, take the conjugate under the modulus of the right hand side in (9).

Remark 3.2. We have also

$$
\begin{aligned}
\left|r_{1} \mathrm{e}^{\mathrm{i} \lambda_{1} x}+r_{2} \mathrm{e}^{\mathrm{i}\left(t+2 \pi d /\left(\lambda_{3}-\lambda_{1}\right)+\lambda_{2} x\right)}+r_{3} \mathrm{e}^{\mathrm{i} \lambda_{3} x}\right| & \\
= & \left|r_{1} \mathrm{e}^{\mathrm{i} \lambda_{1}(x-v)}+r_{2} \mathrm{e}^{\mathrm{i}\left(t+\lambda_{2}(x-v)\right)}+r_{3} \mathrm{e}^{\mathrm{i} \lambda_{3}(x-v)}\right|
\end{aligned}
$$

for all $x$ and $t$, where $v$ satisfies $\lambda_{1} v=2 \pi d /\left(\lambda_{3}-\lambda_{1}\right)+\lambda_{2} v=\lambda_{3} v$ modulo $2 \pi$, that is

$$
v=\frac{2 m \pi}{\lambda_{3}-\lambda_{1}} \quad \text { with } m \text { an inverse of } \frac{\lambda_{3}-\lambda_{2}}{d} \text { modulo } \frac{\lambda_{3}-\lambda_{1}}{d} .
$$




\section{The frequencies of a trigonometric trinomial}

We may suppose without loss of generality that $\lambda_{1}<\lambda_{2}<\lambda_{3}$. Let $k=\left(\lambda_{2}-\lambda_{1}\right) /$ $d$ and $l=\left(\lambda_{3}-\lambda_{2}\right) / d$. Then

$$
\left|r_{1} \mathrm{e}^{\mathrm{i} \lambda_{1} x}+r_{2} \mathrm{e}^{\mathrm{i}\left(t+\lambda_{2} x\right)}+r_{3} \mathrm{e}^{\mathrm{i} \lambda_{3} x}\right|=\left|r_{1} \mathrm{e}^{-\mathrm{i} k(d x)}+r_{2} \mathrm{e}^{\mathrm{i} t}+r_{3} \mathrm{e}^{\mathrm{i} l(d x)}\right| .
$$

A homothety by $d^{-1}$ permits to restrain our study to the function

$$
f(t, x)=\left|r_{1} \mathrm{e}^{-\mathrm{i} k x}+r_{2} \mathrm{e}^{\mathrm{i} t}+r_{3} \mathrm{e}^{\mathrm{i} l x}\right|^{2}
$$

for $x \in \mathbb{R}$ with $k$ and $l$ two positive coprime numbers and $t \in[0, \pi /(k+l)]$. We have

$$
\begin{aligned}
f(-t, x) & =f(t,-x) \\
f(t+2 \pi /(k+l), x) & =f(t, x-2 m \pi /(k+l))
\end{aligned}
$$

for all $x$ and $t$, where $m$ is the inverse of $l$ modulo $k+l$. In particular, if $t=\pi /(k+l)$, we have the symmetry relation

$$
f(\pi /(k+l), x)=f(\pi /(k+l), 2 m \pi /(k+l)-x) .
$$

\section{Location of the maximum point}

The purpose of our first proposition is to deduce a small interval on which a trigonometric trinomial attains its maximum modulus. Note that a trigonometric binomial attains its maximum modulus at a point that depends only on the phase of its coefficients:

- $\left|r_{1} \mathrm{e}^{-\mathrm{i} k x}+r_{2} \mathrm{e}^{\mathrm{i} t}\right|$ attains its maximum at $-t / k$ independently of $r_{1}$ and $r_{2}$,

- $\left|r_{1} \mathrm{e}^{-\mathrm{i} k x}+r_{3} \mathrm{e}^{\mathrm{i} l x}\right|$ attains its maximum at 0 independently of $r_{1}$ and $r_{3}$,

- $\left|r_{2} \mathrm{e}^{\mathrm{i} t}+r_{3} \mathrm{e}^{\mathrm{i} l x}\right|$ attains its maximum at $t / l$ independently of $r_{2}$ and $r_{3}$.

The next proposition shows that if the point at which a trigonometric trinomial attains its maximum modulus changes with the intensity of its coefficients, it changes very little; we get bounds for this point that are independent of the intensities.

Proposition 5.1. Let $k, l$ be two positive coprime integers. Let $r_{1}, r_{2}$ and $r_{3}$ be three positive real numbers. Let $t \in[0, \pi /(k+l)]$. Let

$$
f(x)=\left|r_{1} \mathrm{e}^{-\mathrm{i} k x}+r_{2} \mathrm{e}^{\mathrm{i} t}+r_{3} \mathrm{e}^{\mathrm{i} l x}\right|^{2}
$$

for $x \in \mathbb{R}$.

(a) The function $f$ attains its absolute maximum in the interval $[-t / k, t / l]$. 
(b) If $f$ attains its absolute maximum at a point $y$ outside of $[-t / k, t / l]$ modulo $2 \pi$, then $t=\pi /(k+l)$ and $2 m \pi /(k+l)-y$ lies in $[-t / k, t / l]$ modulo $2 \pi$, where $m$ is the inverse of $l$ modulo $k+l$.

Proof. (a). We have

$$
\begin{aligned}
& f(x)=r_{1}^{2}+r_{2}^{2}+r_{3}^{2}+2 . \\
& \quad\left(r_{1} r_{2} \cos (t+k x)+r_{1} r_{3} \cos ((k+l) x)+r_{2} r_{3} \cos (t-l x)\right) .
\end{aligned}
$$

Let us prove that $f$ attains its absolute maximum on $[-t / k, t / l]$. Let $y$ be outside of $[-t / k, t / l]$ modulo $2 \pi$. Let $I$ be the set of all $x \in[-t / k, t / l]$ such that

$$
\left\{\begin{aligned}
\cos (t+k x) & \geqslant \cos (t+k y) \\
\cos ((k+l) x) & \geqslant \cos ((k+l) y) \\
\cos (t-l x) & \geqslant \cos (t-l y)
\end{aligned}\right.
$$

Note that if $x \in[-t / k, t / l]$, then

$$
\left\{\begin{aligned}
t+k x & \in[0,(k+l) t / l] \\
(k+l) x & \in[-(k+l) t / k,(k+l) t / l] \\
t-l x & \in[0,(k+l) t / k],
\end{aligned}\right.
$$

and that $(k+l) t / k,(k+l) t / l \subset[0, \pi]$. Let

- $\alpha$ be the distance of $t / k+y$ to $(2 \pi / k) \mathbb{Z}$,

- $\beta$ be the distance of $y$ to $(2 \pi /(k+l)) \mathbb{Z}$,

- $\gamma$ be the distance of $t / l-y$ to $(2 \pi / l) \mathbb{Z}$.

Then

$$
I=[-t / k, t / l] \cap[-t / k-\alpha,-t / k+\alpha] \cap[-\beta, \beta] \cap[t / l-\gamma, t / l+\gamma] .
$$

Let us check that $I$ is the nonempty interval

$$
I=[\max (-t / k,-\beta, t / l-\gamma), \min (t / l,-t / k+\alpha, \beta)] .
$$

In fact, we have the following triangular inequalities:

- $-\beta \leqslant-t / k+\alpha$ because $t / k$ is the distance of $(t / k+y)-y$ to $(2 \pi / k(k+l)) \mathbb{Z}$;

- $t / l-\gamma \leqslant-t / k+\alpha$ because $t / l+t / k$ is the distance of $(t / k+y)+(t / l-y)$ to $(2 \pi / k l) \mathbb{Z}$;

- $t / l-\gamma \leqslant \beta$ because $t / l$ is the distance of $(t / l-y)+y$ to $(2 \pi / l(k+l)) \mathbb{Z}$. 
The other six inequalities that are necessary to deduce (15) from (14) are obvious.

(b). We have proved in $(a)$ that there is an $x \in[-t / k, t / l]$ such that $\cos (t+$ $k x) \geqslant \cos (t+k y), \cos ((k+l) x) \geqslant \cos ((k+l) y)$ and $\cos (t-l x) \geqslant \cos (t-l y)$. In fact, at least one of these inequalities is strict unless there are signs $\delta, \epsilon, \eta \in$ $\{-1,1\}$ such that $t+k x=\delta(t+k y), t-l x=\epsilon(t-l y)$ and $(k+l) x=\eta(k+l) y$ modulo $2 \pi$. Two out of these three signs are equal and the corresponding two equations imply the third one with the same sign. This system is therefore equivalent to

$$
\left\{\begin{array} { c } 
{ k ( x - y ) = 0 } \\
{ l ( x - y ) = 0 }
\end{array} \text { or } \quad \left\{\begin{array}{c}
k(x+y)=-2 t \\
l(x+y)=2 t
\end{array}\right.\right.
$$

modulo $2 \pi$. The first pair of equations yields $x=y$ modulo $2 \pi$ because $k$ and $l$ are coprime. Let $m$ be an inverse of $l$ modulo $k+l$; then the second pair of equations is equivalent to

$$
\left\{\begin{aligned}
2(k+l) t & =0 \\
x+y & =2 m t
\end{aligned}\right.
$$

modulo $2 \pi$. Therefore $g$ does not attain its absolute maximum at $y$ unless $t=\pi /(k+l)$ and $2 m \pi /(k+l)-y \in[-t / k, t / l]$.

Remark 5.2. This proposition is a complex version of [16, Lemma 2.1].

\section{Uniqueness of the maximum point}

Note that

$$
\begin{aligned}
r_{1} \mathrm{e}^{-\mathrm{i} k x}+r_{2} \mathrm{e}^{\mathrm{i} t}+r_{3} \mathrm{e}^{\mathrm{i} l x} & =r_{3} \mathrm{e}^{-\mathrm{i} l(-x)}+r_{2} \mathrm{e}^{\mathrm{i} t}+r_{1} \mathrm{e}^{\mathrm{i} k(-x)} \\
& =r_{1}^{\prime} \mathrm{e}^{-\mathrm{i} k^{\prime} x^{\prime}}+r_{2} \mathrm{e}^{\mathrm{i} t}+r_{3}^{\prime} \mathrm{e}^{\mathrm{i} l^{\prime} x^{\prime}}
\end{aligned}
$$

with $r_{1}^{\prime}=r_{3}, r_{3}^{\prime}=r_{1}, k^{\prime}=l, l^{\prime}=k$ and $x^{\prime}=-x$. We may therefore suppose without loss of generality that $k r_{1} \leqslant l r_{3}$.

Our second proposition studies the points at which a trigonometric trinomial attains its maximum modulus. Note that if $k=l=1$, the derivative of $|f|^{2}$ has at most 4 zeroes, so that the modulus of $f$ has at most two maxima and attains its absolute maximum in at most two points. Proposition 6.1 shows that this is true in general, and that if it may attain its absolute maximum in two points, it is so only because of the symmetry given by (12).

Proposition 6.1. Let $k, l$ be two positive coprime integers. Let $r_{1}, r_{2}$ and $r_{3}$ be three positive real numbers such that $k r_{1} \leqslant l r_{3}$. Let $\left.\left.t \in\right] 0, \pi /(k+l)\right]$. Let

$$
f(x)=\left|r_{1} \mathrm{e}^{-\mathrm{i} k x}+r_{2} \mathrm{e}^{\mathrm{i} t}+r_{3} \mathrm{e}^{\mathrm{i} l x}\right|^{2}
$$

for $x \in[-t / k, t / l]$. 
(a) There is a point $x^{*} \in[0, t / l]$ such that $\mathrm{d} f / \mathrm{d} x>0$ on $]-t / k, x^{*}[$ and $\mathrm{d} f / \mathrm{d} x<0$ on $] x^{*}, t / l[$.

(b) There are three cases:

1. $f$ attains its absolute maximum at 0 if and only if $k r_{1}=l r_{3}$;

2. $f$ attains its absolute maximum at $t / l$ if and only if $l=1, t=$ $\pi /(k+1)$ and $k^{2} r_{1} r_{2}+(k+1)^{2} r_{1} r_{3}-r_{2} r_{3} \leqslant 0 ;$

3. otherwise, $f$ attains its absolute maximum in $] 0, t / l[$.

(c) The function $f$ attains its absolute maximum with multiplicity 2 unless $l=1, t=\pi /(k+1)$ and $k^{2} r_{1} r_{2}+(k+1)^{2} r_{1} r_{3}-r_{2} r_{3}=0$, in which case it attains its absolute maximum at $\pi /(k+1)$ with multiplicity 4 .

Proof. (a). By Proposition 5.1 the derivative of $f$ has a zero in $[-t / k, t / l]$. Let us study the sign of the derivative of $f$. Equation (13) yields

$$
\frac{1}{2} \frac{\mathrm{d} f}{\mathrm{~d} x}(x)=-k r_{1} r_{2} \sin (t+k x)-(k+l) r_{1} r_{3} \sin ((k+l) x)+l r_{2} r_{3} \sin (t-l x) .
$$

We wish to compare $\sin (t+k x)$ with $\sin (t-l x)$ : note that

$$
\sin (t+k x)-\sin (t-l x)=2 \sin ((k+l) x / 2) \cos (t+(k-l) x / 2) .
$$

Suppose that $x \in[-t / k, t / l]$. Then

$$
\begin{gathered}
-\pi \leqslant-\pi / k \leqslant-(k+l) t / k \leqslant(k+l) x \leqslant(k+l) t / l \leqslant \pi / l \leqslant \pi \\
0 \leqslant 2 t+(k-l) x \leqslant \begin{cases}2 t+(l-k) t / k=(k+l) t / k & \text { if } k \leqslant l \\
2 t+(k-l) t / l=(k+l) t / l & \text { if } l \leqslant k\end{cases}
\end{gathered}
$$

Suppose that $x \in[-t / k, 0[$ : then it follows that $\sin (t+k x) \leqslant \sin (t-l x)$ and $\sin ((k+l) x) \leqslant 0$ with equality if and only if $k=1$ and $-x=t=\pi /(1+l)$. This yields with $k r_{1} \leqslant l r_{3}$ that

$$
\frac{1}{2} \frac{\mathrm{d} f}{\mathrm{~d} x}(x) \geqslant-(k+l) r_{1} r_{3} \sin ((k+l) x) \geqslant 0
$$

with equality if and only if $k=1$ and $-x=t=\pi /(1+l)$.

If $l \geqslant 2$ and $x \in[0, t / l]$, then

$$
\left\{\begin{aligned}
t+k x & \in[t,(k+l) t / l] \subset[t, \pi / 2] \\
(k+l) x & \in[0,(k+l) t / l] \subset[t, \pi / 2] \\
t-l x & \in[0, t] \subset[0, \pi / 3],
\end{aligned}\right.
$$

so that the second derivative of $f$ is strictly negative on $[0, t / l]$ : its derivative is strictly decreasing on this interval and $(a)$ is proved. Suppose that $l=1$ and 
consider $g(x)=f(t-x)$ for $x \in[0, t]$ : we have to prove that there is a point $x^{*}$ such that $\mathrm{d} g / \mathrm{d} x>0$ on $] 0, x^{*}[$ and $\mathrm{d} g / \mathrm{d} x<0$ on $] x^{*}, t[$. Put $\alpha=(k+1) t$ : then

$$
\frac{1}{2} \frac{\mathrm{d} g}{\mathrm{~d} x}(x)=k r_{1} r_{2} \sin (\alpha-k x)+(k+1) r_{1} r_{3} \sin (\alpha-(k+1) x)-r_{2} r_{3} \sin x
$$

and it suffices to prove that

$$
\frac{1}{2 \sin x} \frac{\mathrm{d} g}{\mathrm{~d} x}(x)=k r_{1} r_{2} \frac{\sin (\alpha-k x)}{\sin x}+(k+1) r_{1} r_{3} \frac{\sin (\alpha-(k+1) x)}{\sin x}-r_{2} r_{3}
$$

decreases strictly with $x \in] 0, \alpha /(k+1)]$. Let us study the sign of

$$
\frac{\mathrm{d}}{\mathrm{d} x} \frac{\sin (\alpha-k x)}{\sin x}=\frac{-k \cos (\alpha-k x) \sin x-\sin (\alpha-k x) \cos x}{\sin ^{2} x}
$$

for $\alpha \in] 0, \pi]$ and $x \in] 0, \alpha / k]$. If $k=1$, then

$$
-k \cos (\alpha-k x) \sin x-\sin (\alpha-k x) \cos x=-\sin \alpha \leqslant 0
$$

and the inequality is strict unless $\alpha=\pi$. Let us prove by induction on $k$ that $k \cos (\alpha-k x) \sin x+\sin (\alpha-k x) \cos x>0$ for all $k \geqslant 2, \alpha \in] 0, \pi]$ and $x \in] 0$, $\alpha / k]$. This will end the proof of $(a)$. Let $k \geqslant 1$ and $x \in] 0, \alpha /(k+1)]$. Then

$$
\begin{aligned}
&(k+1) \cos (\alpha-(k+1) x) \sin x+\sin (\alpha-(k+1) x) \cos x \\
&=(k+1) \cos (\alpha-k x) \cos x \sin x+(k+1) \sin (\alpha-k x) \sin ^{2} x \\
& \quad+\sin (\alpha-k x) \cos ^{2} x-\cos (\alpha-k x) \sin x \cos x \\
&=(k \cos (\alpha-k x) \sin x+\sin (\alpha-k x) \cos x) \cos x \\
& \quad+(k+1) \sin (\alpha-k x) \sin ^{2} x \\
& \geqslant(k+1) \sin (\alpha-k x) \sin ^{2} x>0
\end{aligned}
$$

(b). 1. By Proposition 5.1 and $(a), f$ attains its absolute maximum at 0 if and only if 0 is a critical point for $f$. We have

$$
\frac{1}{2} \frac{\mathrm{d} f}{\mathrm{~d} x}(0)=\left(l r_{3}-k r_{1}\right) r_{2} \sin t \geqslant 0
$$

and equality holds if and only if $k r_{1}=l r_{3}$.

2. We have

$$
\frac{1}{2} \frac{\mathrm{d} f}{\mathrm{~d} x}(t / l)=\left(-k r_{1} r_{2}-(k+l) r_{1} r_{3}\right) \sin ((k+l) t / l) \leqslant 0
$$

and equality holds if and only if $l=1$ and $t=\pi /(k+1)$. Let $l=1$ and $t=\pi /(k+1)$ and let us use the notation introduced in the last part of the proof of $(a)$ : we need to characterise the case that $g$ has a maximum at 0 . As $\alpha=\pi$ and

$$
\frac{1}{2 \sin x} \frac{\mathrm{d} g}{\mathrm{~d} x}(x)=k^{2} r_{1} r_{2}+(k+1)^{2} r_{1} r_{3}-r_{2} r_{3}+o(x)
$$


decreases strictly with $x \in] 0, \pi /(k+1)], g$ has a maximum at 0 if and only if $k^{2} r_{1} r_{2}+(k+1)^{2} r_{1} r_{3}-r_{2} r_{3} \leqslant 0$.

$(c)$. If $l \geqslant 2$, then the second derivative of $f$ is strictly negative on $[0, t / l]$. If $l=1$, then the derivative of (18) is strictly negative on $] 0, \alpha /(k+1)]$ : this yields that the second derivative of $g$ can only vanish at 0 . By $(b) 2, g$ has a maximum at 0 only if $t=\pi /(k+1)$; then

$$
\begin{gathered}
\frac{1}{2} \frac{\mathrm{d}^{2} g}{\mathrm{~d} x^{2}}(0)=k^{2} r_{1} r_{2}+(k+1)^{2} r_{1} r_{3}-r_{2} r_{3} \\
\frac{1}{2} \frac{\mathrm{d}^{4} g}{\mathrm{~d} x^{4}}(0)=-k^{4} r_{1} r_{2}-(k+1)^{4} r_{1} r_{3}+r_{2} r_{3}
\end{gathered}
$$

If (20) vanishes, then the sum of (21) with (20) yields

$$
\frac{1}{2} \frac{\mathrm{d}^{4} g}{\mathrm{~d} x^{4}}(0)=-k(k+1) r_{1}\left((k-1) k r_{2}+(k+1)(k+2) r_{3}\right)<0 .
$$

Remark 6.2. We were able to prove directly that the system

$$
\left\{\begin{array}{l}
f(x)=f(y) \\
\frac{\mathrm{d} f}{\mathrm{~d} x}(x)=\frac{\mathrm{d} f}{\mathrm{~d} x}(y)=0 \\
\frac{\mathrm{d}^{2} f}{\mathrm{~d} x^{2}}(x), \frac{\mathrm{d}^{2} f}{\mathrm{~d} x^{2}}(y) \leqslant 0
\end{array}\right.
$$

implies $x=y$ modulo $2 \pi$ or $t=\pi /(k+l)$ and $x+y=2 m \pi /(k+l)$, but our computations are very involved and opaque.

Remark 6.3. Suppose that $l=k=1$. If $t \in] 0, \pi / 2[$, it is necessary to solve a generally irreducible quartic equation in order to compute the maximum of $f$. If $t=\pi / 2$, it suffices to solve a linear equation and one gets the following expression for $\max _{x}\left|r_{1} \mathrm{e}^{-\mathrm{i} x}+\mathrm{i} r_{2}+r_{3} \mathrm{e}^{\mathrm{i} x}\right|:$

$$
\begin{cases}\left(r_{1}+r_{3}\right) \sqrt{1+r_{2}^{2} / 4 r_{1} r_{3}} & \text { if }\left|r_{1}^{-1}-r_{3}^{-1}\right|<4 r_{2}^{-1} \\ r_{2}+\left|r_{3}-r_{1}\right| & \text { otherwise. }\end{cases}
$$

In the first case, the maximum is attained at the two points $x^{*} \operatorname{such}$ that $\sin x^{*}=$ $r_{2}\left(r_{3}-r_{1}\right) / 4 r_{1} r_{3}$.

Remark 6.4. Suppose that $l=1$ and $k=2$. If $t \in] 0, \pi / 3[$, it is necessary to solve a generally irreducible sextic equation in order to compute the maximum of $f$. If $t=\pi / 3$, it suffices to solve a quadratic equation and one gets the following expression for $\max _{x}\left|r_{1} \mathrm{e}^{-\mathrm{i} 2 x}+r_{2} \mathrm{e}^{\mathrm{i} \pi / 3}+r_{3} \mathrm{e}^{\mathrm{i} x}\right|:$ if $r_{1}^{-1}-4 r_{3}^{-1}<9 r_{2}^{-1}$, then its square makes

$$
r_{1}^{2}+\frac{2}{3} r_{2}^{2}+r_{3}^{2}+r_{1} r_{2}+2 r_{1} r_{3}\left[\left(\left(\frac{r_{2}}{3 r_{3}}\right)^{2}+\frac{r_{2}}{3 r_{1}}+1\right)^{3 / 2}-\left(\frac{r_{2}}{3 r_{3}}\right)^{3}\right]
$$


and the maximum is attained at the two points $x^{*}$ such that

$$
2 \cos \left(\pi / 3-x^{*}\right)=\left(\left(\frac{r_{2}}{3 r_{3}}\right)^{2}+\frac{r_{2}}{3 r_{1}}+1\right)^{1 / 2}-\frac{r_{2}}{3 r_{3}}
$$

otherwise, it makes $-r_{1}+r_{2}+r_{3}$.

\section{The maximum modulus points of a trigono- metric trinomial}

If we undo all the reductions that we did in Sections 3 and 4 and in the beginning of Section 6, we get the following theorem.

Theorem 7.1. Let $\lambda_{1}, \lambda_{2}$ and $\lambda_{3}$ be three pairwise distinct integers such that $\lambda_{2}$ is between $\lambda_{1}$ and $\lambda_{3}$. Let $r_{1}, r_{2}$ and $r_{3}$ be three positive real numbers. Given three real numbers $t_{1}, t_{2}$ and $t_{3}$, consider the trigonometric trinomial

$$
T(x)=r_{1} \mathrm{e}^{\mathrm{i}\left(t_{1}+\lambda_{1} x\right)}+r_{2} \mathrm{e}^{\mathrm{i}\left(t_{2}+\lambda_{2} x\right)}+r_{3} \mathrm{e}^{\mathrm{i}\left(t_{3}+\lambda_{3} x\right)}
$$

for $x \in \mathbb{R}$. Let $d=\operatorname{gcd}\left(\lambda_{2}-\lambda_{1}, \lambda_{3}-\lambda_{2}\right)$ and choose integers $a_{1}$ and $a_{3}$ such that

$$
\tau=\frac{\lambda_{2}-\lambda_{3}}{d}\left(t_{1}-2 \pi a_{1}\right)+\frac{\lambda_{3}-\lambda_{1}}{d} t_{2}+\frac{\lambda_{1}-\lambda_{2}}{d}\left(t_{3}-2 \pi a_{3}\right),
$$

satisfies $|\tau| \leqslant \pi$. Let $\tilde{t}_{1}=t_{1}-2 \pi a_{1}$ and $\tilde{t}_{3}=t_{3}-2 \pi a_{3}$.

(a) The trigonometric trinomial $T$ attains its maximum modulus at a unique point of the interval bounded by $\left(\tilde{t}_{1}-t_{2}\right) /\left(\lambda_{2}-\lambda_{1}\right)$ and $\left(t_{2}-\tilde{t}_{3}\right) /\left(\lambda_{3}-\lambda_{2}\right)$. More precisely,

- if $r_{1}\left|\lambda_{2}-\lambda_{1}\right| \leqslant r_{3}\left|\lambda_{3}-\lambda_{2}\right|$, then this point is between $\left(\tilde{t}_{1}-\tilde{t}_{3}\right) /\left(\lambda_{3}-\lambda_{1}\right)$ and $\left(t_{2}-\tilde{t}_{3}\right) /\left(\lambda_{3}-\lambda_{2}\right)$

- if $r_{1}\left|\lambda_{2}-\lambda_{1}\right| \geqslant r_{3}\left|\lambda_{3}-\lambda_{2}\right|$, then this point is between $\left(\tilde{t}_{1}-\tilde{t}_{3}\right) /\left(\lambda_{3}-\lambda_{1}\right)$ and $\left(\tilde{t}_{1}-t_{2}\right) /\left(\lambda_{2}-\lambda_{1}\right)$

- $T$ attains its maximum modulus at $\left(\tilde{t}_{1}-\tilde{t}_{3}\right) /\left(\lambda_{3}-\lambda_{1}\right)$ if and only if $r_{1}\left|\lambda_{2}-\lambda_{1}\right|=r_{3}\left|\lambda_{3}-\lambda_{2}\right|$ or $\tau=0$.

(b) The function $T$ attains its maximum modulus at a unique point modulo $2 \pi / d$, and with multiplicity 2 , unless $|\tau|=\pi$.

(c) Suppose that $|\tau|=\pi$, i. e.,

$$
\frac{\lambda_{2}-\lambda_{3}}{d} t_{1}+\frac{\lambda_{3}-\lambda_{1}}{d} t_{2}+\frac{\lambda_{1}-\lambda_{2}}{d} t_{3}=\pi \bmod 2 \pi
$$

Let $s$ be a solution to $2 t_{1}+\lambda_{1} s=2 t_{2}+\lambda_{2} s=2 t_{3}+\lambda_{3} s$ modulo $2 \pi$ : $s$ is unique modulo $2 \pi / d$. Then $T(s-x)=\mathrm{e}^{\mathrm{i}\left(2 t_{2}+\lambda_{2} s\right)} \overline{T(x)}$ for all $x$. Suppose that $\left|\lambda_{3}-\lambda_{2}\right| \leqslant\left|\lambda_{2}-\lambda_{1}\right|$. There are three cases. 
1. If $\lambda_{2}-\lambda_{1}=k\left(\lambda_{3}-\lambda_{2}\right)$ with $k \geqslant 2$ integer and

$$
r_{1}^{-1}-k^{2} r_{3}^{-1} \geqslant(k+1)^{2} r_{2}^{-1},
$$

then $T$ attains its maximum modulus, $-r_{1}+r_{2}+r_{3}$, only at $x=$ $\left(t_{2}-\tilde{t}_{3}\right) /\left(\lambda_{3}-\lambda_{2}\right)$ modulo $2 \pi / d$, with multiplicity 2 if the inequality is strict and with multiplicity 4 if there is equality;

2. if $\lambda_{2}-\lambda_{1}=\lambda_{3}-\lambda_{2}$ and

$$
\left|r_{1}^{-1}-r_{3}^{-1}\right| \geqslant 4 r_{2}^{-1}
$$

then $T$ attains its maximum modulus, $r_{2}+\left|r_{3}-r_{1}\right|$, at a unique point $x$ modulo $2 \pi / d$, with multiplicity 2 if the inequality is strict and with multiplicity 4 if there is equality. This point is $\left(t_{2}-\tilde{t}_{3}\right) /\left(\lambda_{3}-\lambda_{2}\right)$ if $r_{1}<r_{3}$, and $\left(\tilde{t}_{1}-t_{2}\right) /\left(\lambda_{2}-\lambda_{1}\right)$ if $r_{3}<r_{1}$;

3. otherwise $T$ attains its maximum modulus at exactly two points $x$ and $y$ modulo $2 \pi / d$, with multiplicity 2 , where $x$ is strictly between $\left(\tilde{t}_{1}-t_{2}\right) /\left(\lambda_{2}-\lambda_{1}\right)$ and $\left(t_{2}-\tilde{t}_{3}\right) /\left(\lambda_{3}-\lambda_{2}\right)$, and $x+y=s$ modulo $2 \pi / d$.

Note that $s-x=x$ modulo $2 \pi / d$ in Cases 1 and 2.

\section{Exposed and extreme points of the unit ball of $\mathscr{C}_{\Lambda}$}

The characterisation of the maximum modulus points of a trigonometric trinomial permits to compute the exposed and the extreme points of the unit ball of $\mathscr{C}_{\Lambda}$. We begin with a lemma.

Lemma 8.1. (a) A trigonometric trinomial with a given spectrum that attains its maximum modulus at two given points modulo $2 \pi / d$ is determined by its value at these points.

(b) The trigonometric trinomials with a given spectrum that attain their maximum modulus with multiplicity 4 at a given point and have a given value at this point lie on a parabola.

Proof. We will use the notation of Theorem 7.1. Without loss of generality, we may suppose that $\lambda_{1}=-k, \lambda_{2}=0$ and $\lambda_{3}=l$ with $k$ and $l$ two positive coprime integers. Let $x$ and $y$ be two real numbers that are different modulo $2 \pi / d$, let $\vartheta$ and $\zeta$ be real numbers and let $\varrho$ be a positive real number.

(a). Let us prove that at most one trigonometric trinomial $T$ attains its maximum modulus at $x$ and $y$ and satisfies $T(x)=\varrho \mathrm{e}^{\mathrm{i} \vartheta}$ and $T(y)=\varrho \mathrm{e}^{\mathrm{i} \zeta}$.

Let us translate $T$ by $(x+y) / 2$ : we may suppose that $x+y=0$. Let us divide $T$ by $\mathrm{e}^{\mathrm{i}(\vartheta+\zeta) / 2}$ : we may suppose that $\vartheta+\zeta=0$. As $T$ attains its maximum modulus at the two points $x$ and $y$, we have $s=x+y=0$ and 
$2 t_{1}-k s=2 t_{2}=2 t_{3}+l s=\vartheta+\zeta=0$ modulo $2 \pi$. Therefore $t_{1}=t_{2}=t_{3}=0$ modulo $\pi$. Let $p_{j}=\mathrm{e}^{\mathrm{i} t_{j}} r_{j}$ : the $p_{j}$ are nonzero real numbers. We have

$$
T(x)=p_{1} \mathrm{e}^{-\mathrm{i} k x}+p_{2}+p_{3} \mathrm{e}^{\mathrm{i} l x}=\varrho \mathrm{e}^{\mathrm{i} \vartheta},
$$

so that, multiplying by $\mathrm{e}^{-\mathrm{i} \vartheta}$ and taking real and imaginary parts,

$$
\begin{gathered}
p_{1} \cos (\vartheta+k x)+p_{3} \cos (\vartheta-l x)=\varrho-p_{2} \cos \vartheta \\
p_{1} \sin (\vartheta+k x)+p_{3} \sin (\vartheta-l x)=-p_{2} \sin \vartheta .
\end{gathered}
$$

The computation

$$
\frac{1}{2} \frac{\mathrm{d}|T|^{2}}{\mathrm{~d} x}(x)=\Re\left(\overline{T(x)} \frac{\mathrm{d} T}{\mathrm{~d} x}(x)\right)=\Re\left(\overline{T(x)}\left(-\mathrm{i} k p_{1} \mathrm{e}^{-\mathrm{i} k x}+\mathrm{i} l p_{3} \mathrm{e}^{\mathrm{i} l x}\right)\right),
$$

yields

$$
k p_{1} \sin (\vartheta+k x)-l p_{3} \sin (\vartheta-l x)=0 .
$$

Equations (25) and (26) yield $p_{1}$ and $p_{3}$ as linear functions of $p_{2}$ because $\sin (\vartheta+$ $k x) \sin (\vartheta-l x) \neq 0$ : otherwise both factors vanish, so that $\vartheta=x=0$ modulo $\pi$ and $x=y$ modulo $2 \pi$. As $\varrho \neq 0$, Equation (24) has at most one solution in $p_{2}$.

(b). We may suppose that $l=1$. Let us determine all trigonometric trinomials $T$ that attain their maximum modulus at $x$ with multiplicity 4 and satisfy $T(x)=\varrho \mathrm{e}^{\mathrm{i} \vartheta}$. Let us translate $T$ by $x$ : we may suppose that $x=0$. Let us divide $T$ by $\mathrm{e}^{\mathrm{i} \vartheta}$ : we may suppose that $\vartheta=0$. As $T$ attains its maximum modulus at 0 with multiplicity 4 , we have $s-0=0$ and $2 t_{1}-k s=2 t_{2}=2 t_{3}+s=2 \vartheta=0$ modulo $2 \pi$. Therefore $t_{1}=t_{2}=t_{3}=0$ modulo $\pi$. Let $p_{j}=\mathrm{e}^{\mathrm{i} t_{j}} r_{j}$ : the $p_{j}$ are nonzero real numbers and satisfy the system

$$
\left\{\begin{array}{l}
p_{1}+p_{2}+p_{3}=\varrho \\
k^{2} p_{1} p_{2}+(k+1)^{2} p_{1} p_{3}+p_{2} p_{3}=0
\end{array}\right.
$$

and therefore

$$
\left(k p_{1}-p_{3}\right)^{2}=\varrho\left(k^{2} p_{1}+p_{3}\right) .
$$

More precisely, Theorem 7.1 $(c)$ yields that if $k \geqslant 2$, the $T$ form a parabolic arc and if $k=1$, the $T$ form two arcs of a parabola.

Remark 8.2. The equality

$$
\max _{x}\left|r_{1} \mathrm{e}^{\mathrm{i}\left(t_{1}+\lambda_{1} x\right)}+r_{2} \mathrm{e}^{\mathrm{i}\left(t_{2}+\lambda_{2} x\right)}\right|=r_{1}+r_{2}
$$

shows that the exposed points of the unit ball of the space $\mathscr{C}_{\left\{\lambda_{1}, \lambda_{2}\right\}}$ are the trigonometric monomials $\mathrm{e}^{\mathrm{i} \alpha} \mathrm{e}_{\lambda_{1}}$ and $\mathrm{e}^{\mathrm{i} \alpha} \mathrm{e}_{\lambda_{2}}$ with $\alpha \in \mathbb{R}$ and that no trigonometric binomial is an extreme point of the unit ball of $\mathscr{C}_{\Lambda}$.

Proof of Theorem 1.2 (a). A linear functional on $\mathscr{C}_{\Lambda}$ extends to a linear functional on the space of continuous functions, that is, to a measure $\mu$, with same norm. A measure $\mu$ attains its norm only at functions with constant modulus 
on its support. If $\mu$ attains its norm on a trigonometric binomial, then it attains its norm on a trigonometric monomial because this trigonometric binomial is a convex combination of two trigonometric monomials with same norm by Equation (27). If $\mu$ attains its norm on a trigonometric trinomial $T$, there are two cases by Theorem $7.1(b, c)$ :

- $T$ attains its maximum modulus at a unique point modulo $2 \pi / d$ : then the support of $\mu$ has only one point modulo $2 \pi / d$, so that $\mu$ is the multiple of a Dirac measure and attains its norm on any trigonometric monomial.

- $T$ attains its maximum modulus at two points modulo $2 \pi / d$.

Conversely, the trigonometric monomial $\mathrm{e}^{\mathrm{i} \alpha} \mathrm{e}_{\lambda}$ is exposed to the linear form

$$
U \mapsto \frac{1}{2 \pi} \int_{0}^{2 \pi} U(x) \mathrm{e}^{-\mathrm{i}(\alpha+\lambda x)} \mathrm{d} x .
$$

A trigonometric trinomial $T$ that attains its maximum modulus, 1, at two points $x_{1}^{*}$ and $x_{2}^{*}$ modulo $2 \pi / d$ is exposed, by Lemma $8.1(a)$, to any nontrivial convex combination of the unimodular multiples of Dirac measures $\overline{T\left(x_{1}^{*}\right)} \delta_{x_{1}^{*}}$ and $\overline{T\left(x_{2}^{*}\right)} \delta_{x_{2}^{*}}$.

Remark 8.3. This is a complex version of [16, Lemma 2.3].

Proof of Theorem 1.2 (b). Let $K$ be the unit ball of $\mathscr{C}_{\Lambda}$. Straszewicz's Theorem 21 tells that the exposed points of $K$ are dense in the set of its extreme points. Let $U$ be a limit point of exposed points of $K$. If $U$ is a trigonometric monomial, $U$ is exposed. If $U$ is a trigonometric binomial, $U$ is not an extreme point of $K$ by Remark 8.2 If $U$ is a trigonometric trinomial, it is the limit point of trigonometric trinomials that attain their maximum modulus twice modulo $2 \pi / d$, so that either $U$ also attains its maximum modulus twice modulo $2 \pi / d$ or, by Rolle's Theorem, $U$ attains its maximum modulus with multiplicity 4. Let us prove that if a trigonometric trinomial $U$ attains its maximum modulus with multiplicity 4 at a point $x$, then $U$ is an extreme point of $K$. Suppose that $U$ is the midpoint of two points $A$ and $B$ in $K$. Then $|A(x)| \leqslant 1,|B(x)| \leqslant 1$ and $(A(x)+B(x)) / 2=U(x)$, so that $A(x)=B(x)=U(x)$. Furthermore

$$
\begin{aligned}
|U(x+h)| & \leqslant \frac{|A(x+h)|+|B(x+h)|}{2} \\
& =1+\frac{h^{2}}{4}\left(\frac{\mathrm{d}^{2}|A|}{\mathrm{d} x^{2}}(x)+\frac{\mathrm{d}^{2}|B|}{\mathrm{d} x^{2}}(x)\right)+o\left(h^{2}\right)
\end{aligned}
$$

so that, as $|U(x+h)|=1+o\left(h^{3}\right)$,

$$
\frac{\mathrm{d}^{2}|A|}{\mathrm{d} x^{2}}(x) \leqslant 0, \frac{\mathrm{d}^{2}|B|}{\mathrm{d} x^{2}}(x) \leqslant 0 \text { and } \frac{\mathrm{d}^{2}|A|}{\mathrm{d} x^{2}}(x)+\frac{\mathrm{d}^{2}|B|}{\mathrm{d} x^{2}}(x) \geqslant 0
$$

and therefore $A$ and $B$ also attain their maximum modulus with multiplicity 4 at $x$. As this implies that $A$ and $B$ are trigonometric trinomials, Lemma $8.1(b)$ yields that $U, A$ and $B$ lie on a parabola: this implies $A=B=U$. 
Remark 8.4. The set of extreme points of the unit ball of $\mathscr{C}_{\Lambda}$ is not closed: for example, if $\lambda_{2}$ is between $\lambda_{1}$ and $\lambda_{3}$, every absolutely convex combination of $\mathrm{e}_{\lambda_{1}}$ and $\mathrm{e}_{\lambda_{3}}$ is a limit point of exposed points.

Remark 8.5. If $\lambda_{1}-\lambda_{2}$ is not a multiple of $\lambda_{3}-\lambda_{2}$, nor vice versa, then we obtain that every extreme point of the unit ball of $\mathscr{C}_{\Lambda}$ is exposed.

Remark 8.6. In particular, compare our description of the extreme points of the unit ball of $\mathscr{C}_{\{0,1,2\}}$ with the characterisation given in [6].

\section{Dependence of the maximum modulus on the arguments}

We wish to study how the maximum modulus of a trigonometric trinomial depends on the phase of its coefficients. We shall use the following formula that gives an expression for the directional derivative of a maximum function. It was established in [3]. Elementary properties of maximum functions are addressed in [15, Part Two, Problems 223-226].

N. G. Chebotarëv's formula ([5, Th. VI.3.2, (3.6)]). Let $I \subset \mathbb{R}$ be an open interval and let $K$ be a compact space. Let $f(t, x)$ be a function on $I \times K$ that is continuous together with $\frac{\partial f}{\partial t}(t, x)$. Let

$$
f^{*}(t)=\max _{x \in K} f(t, x) .
$$

Then $f^{*}(t)$ admits the following expansion at every $t \in I$ :

$$
f^{*}(t+h)=f^{*}(t)+\max _{f(t, x)=f^{*}(t)}\left(h \frac{\partial f}{\partial t}(t, x)\right)+o(h) .
$$

Proposition 9.1. Let $k, l$ be two positive coprime integers. Let $r_{1}, r_{2}$ and $r_{3}$ be three positive real numbers. Then

$$
\max _{x}\left|r_{1} \mathrm{e}^{-\mathrm{i} k x}+r_{2} \mathrm{e}^{\mathrm{i} t}+r_{3} \mathrm{e}^{\mathrm{i} l x}\right|
$$

is an even $2 \pi /(k+l)$-periodic function of that strictly decreases on $[0, \pi /(k+l)]$ : in particular

$$
\min _{t} \max _{x}\left|r_{1} \mathrm{e}^{-\mathrm{i} k x}+r_{2} \mathrm{e}^{\mathrm{i} t}+r_{3} \mathrm{e}^{\mathrm{i} l x}\right|=\max _{x}\left|r_{1} \mathrm{e}^{-\mathrm{i} k x}+r_{2} \mathrm{e}^{\mathrm{i} \pi /(k+l)}+r_{3} \mathrm{e}^{\mathrm{i} l x}\right| .
$$

Proof. Let

$$
f(t, x)=\left|r_{1} \mathrm{e}^{-\mathrm{i} k x}+r_{2} \mathrm{e}^{\mathrm{i} t}+r_{3} \mathrm{e}^{\mathrm{i} l x}\right|^{2} .
$$

By (10) and (11), $f^{*}$ is an even $2 \pi /(k+l)$-periodic function.

Let $t \in] 0, \pi /(k+l)\left[\right.$ and choose $x^{*}$ such that $f\left(t, x^{*}\right)=f^{*}(t)$ : then $x^{*} \in$ $[-t / k, t / l]$ by Proposition 5.1, so that

$$
\frac{1}{2 r_{2}} \frac{\partial f}{\partial t}\left(t, x^{*}\right)=-r_{1} \sin \left(t+k x^{*}\right)-r_{3} \sin \left(t-l x^{*}\right)<0
$$


because $t+k x^{*} \in[0,(k+l) t / l]$ and $t-l x^{*} \in[0,(k+l) t / k]$ do not vanish simultaneously. By Formula (28), $f^{*}$ decreases strictly on $[0, \pi /(k+l)]$.

Proposition 9.2. Let $k, l$ be two positive coprime integers. Let $r_{1}, r_{2}$ and $r_{3}$ be three positive real numbers. Then

$$
\frac{\max _{x}\left|r_{1} \mathrm{e}^{-\mathrm{i} k x}+r_{2} \mathrm{e}^{\mathrm{i} t}+r_{3} \mathrm{e}^{\mathrm{i} l x}\right|}{\left|r_{1}+r_{2} \mathrm{e}^{\mathrm{i} t}+r_{3}\right|}
$$

is an increasing function of $t \in[0, \pi /(k+l)]$. If $k r_{1}=l r_{3}$, it is constantly equal to 1 ; otherwise it is strictly increasing.

Proof. Let $f(t, x)$ be as in (29): then the expression (30) is $g^{*}(t)^{1 / 2}$ with

$$
g(t, x)=\frac{f(t, x)}{f(t, 0)}
$$

If $k r_{1}=l r_{3}$, then $f(t, 0)=f^{*}(t)$, so that $g^{*}(t)=1$. As shown in the beginning of Section [6. we may suppose without loss of generality that $k r_{1}<l r_{3}$. Let $t \in] 0, \pi /(k+l)\left[\right.$ and choose $x^{*}$ such that $f\left(t, x^{*}\right)=f^{*}(t)$ : then $\left.x^{*} \in\right] 0, t / l[$ by Propositions 5.1 and 6.1 and

$$
\begin{aligned}
\frac{f(t, 0)^{2}}{2 r_{2}} \frac{\partial g}{\partial t} & \left(t, x^{*}\right)=\frac{1}{2 r_{2}}\left(\frac{\partial f}{\partial t}\left(t, x^{*}\right) f(t, 0)-f\left(t, x^{*}\right) \frac{\partial f}{\partial t}(t, 0)\right) \\
& =\left(-r_{1} \sin \left(t+k x^{*}\right)-r_{3} \sin \left(t-l x^{*}\right)\right) f(t, 0)+f^{*}(t)\left(r_{1}+r_{3}\right) \sin t \\
& =h(0) f^{*}(t)-h\left(x^{*}\right) f(t, 0)
\end{aligned}
$$

with

$$
h(x)=r_{1} \sin (t+k x)+r_{3} \sin (t-l x) .
$$

Let us show that $h$ is strictly decreasing on $[0, t / l]$ : in fact, if $x \in] 0, t / l[$,

$$
\frac{\mathrm{d} h}{\mathrm{~d} x}(x)=k r_{1} \cos (t+k x)-l r_{3} \cos (t-l x)<\left(k r_{1}-l r_{3}\right) \cos (t-l x)<0 .
$$

As $f^{*}(t)>f(t, 0)$ and $h(0)>h\left(x^{*}\right),(\partial g / \partial t)\left(t, x^{*}\right)>0$. By N. G. Chebotarëv's formula, $g^{*}$ increases strictly on $[0, \pi /(k+l)]$.

It is possible to describe the decrease of the maximum modulus of a trigonometric trinomial independently of the $r$ 's as follows.

Proposition 9.3. Let $k$ and $l$ be two positive coprime integers. Let $r_{1}, r_{2}$ and $r_{3}$ be three positive real numbers. Let $0 \leqslant t^{\prime}<t \leqslant \pi /(k+l)$. Then

$$
\max _{x}\left|r_{1} \mathrm{e}^{-\mathrm{i} k x}+r_{2} \mathrm{e}^{\mathrm{i} t^{\prime}}+r_{3} \mathrm{e}^{\mathrm{i} l x}\right| \leqslant \frac{\cos \left(t^{\prime} / 2\right)}{\cos (t / 2)} \max _{x}\left|r_{1} \mathrm{e}^{-\mathrm{i} k x}+r_{2} \mathrm{e}^{\mathrm{i} t}+r_{3} \mathrm{e}^{\mathrm{i} l x}\right|
$$

with equality if and only if $r_{1}: r_{2}: r_{3}=l: k+l: k$. 
Proof. Let us apply Proposition 9.2, We have

$$
\begin{aligned}
\frac{\left|r_{1}+r_{2} \mathrm{e}^{\mathrm{i} t^{\prime}}+r_{3}\right|^{2}}{\left|r_{1}+r_{2} \mathrm{e}^{\mathrm{i} t}+r_{3}\right|^{2}} & =1+\frac{2 r_{2}\left(r_{1}+r_{3}\right)\left(\cos t^{\prime}-\cos t\right)}{\left(r_{1}+r_{3}\right)^{2}+2 r_{2}\left(r_{1}+r_{3}\right) \cos t+r_{2}^{2}} \\
& =1+\frac{\cos t^{\prime}-\cos t}{\cos t+\left(r_{2}^{2}+\left(r_{1}+r_{3}\right)^{2}\right) / 2 r_{2}\left(r_{1}+r_{3}\right)} \\
& \leqslant 1+\frac{\cos t^{\prime}-\cos t}{\cos t+1}=\frac{\cos t^{\prime}+1}{\cos t+1}
\end{aligned}
$$

by the arithmetic-geometric inequality, with equality if and only if $r_{2}=r_{1}+r_{3}$. Therefore Inequality (31) holds, with equality if and only if $k r_{1}=l r_{3}$ and $r_{2}=r_{1}+r_{3}$.

We may now find the minimum of the maximum modulus of a trigonometric trinomial with fixed Fourier coefficient arguments and moduli sum. Proposition 9.3 yields with $t^{\prime}=0$

Corollary 9.4. Let $k$ and $l$ be two positive coprime integers. Let $r_{1}, r_{2}$ and $r_{3}$ be three positive real numbers. Let $t \in] 0, \pi /(k+l)]$. Then

$$
\frac{\max _{x}\left|r_{1} \mathrm{e}^{-\mathrm{i} k x}+r_{2} \mathrm{e}^{\mathrm{i} t}+r_{3} \mathrm{e}^{\mathrm{i} l x}\right|}{r_{1}+r_{2}+r_{3}} \geqslant \cos (t / 2)
$$

with equality if and only if $r_{1}: r_{2}: r_{3}=l: k+l: k$.

Remark 9.5. There is a shortcut proof of Corollary 9.4 .

$$
\begin{aligned}
\frac{\max _{x}\left|r_{1} \mathrm{e}^{-\mathrm{i} k x}+r_{2} \mathrm{e}^{\mathrm{i} t}+r_{3} \mathrm{e}^{\mathrm{i} l x}\right|}{r_{1}+r_{2}+r_{3}} & \geqslant \frac{\left|r_{1}+r_{2} \mathrm{e}^{\mathrm{i} t}+r_{3}\right|}{r_{1}+r_{2}+r_{3}} \\
& =\sqrt{1-\frac{4\left(r_{1}+r_{3}\right) r_{2}}{\left(r_{1}+r_{2}+r_{3}\right)^{2}} \sin ^{2}(t / 2)} \\
& \geqslant \sqrt{1-\sin ^{2}(t / 2)}=\cos (t / 2)
\end{aligned}
$$

and equality holds if and only if $\left|r_{1} \mathrm{e}^{-\mathrm{i} k x}+r_{2} \mathrm{e}^{\mathrm{i} t}+r_{3} \mathrm{e}^{\mathrm{i} l x}\right|$ is maximal for $x=0$ and $r_{1}+r_{3}=r_{2}$.

\section{The norm of unimodular relative Fourier multipliers}

We may now compute the norm of unimodular relative Fourier multipliers.

Corollary 10.1. Let $k$ and $l$ be two positive coprime integers. Let $t \in[0, \pi /$ $(k+l)]$. Let $M$ be the relative Fourier multiplier $(0, t, 0)$ that maps the element

$$
r_{1} \mathrm{e}^{\mathrm{i} u_{1}} \mathrm{e}_{-k}+r_{2} \mathrm{e}^{\mathrm{i} u_{2}} \mathrm{e}_{0}+r_{3} \mathrm{e}^{\mathrm{i} u_{3}} \mathrm{e}_{l}
$$


of the normed space $\mathscr{C}_{\{-k, 0, l\}}$ on

$$
r_{1} \mathrm{e}^{\mathrm{i} u_{1}} \mathrm{e}_{-k}+r_{2} \mathrm{e}^{\mathrm{i}\left(t+u_{2}\right)} \mathrm{e}_{0}+r_{3} \mathrm{e}^{\mathrm{i} u_{3}} \mathrm{e}_{l} .
$$

Then $M$ has norm $\cos (\pi / 2(k+l)-t / 2) / \cos (\pi / 2(k+l))$ and attains its norm exactly at elements of form (32) with $r_{1}: r_{2}: r_{3}=l: k+l: k$ and

$$
-l u_{1}+(k+l) u_{2}-k u_{3}=\pi \bmod 2 \pi .
$$

Proof. This follows from Proposition 9.3 and the concavity of $\cos$ on $[0, \pi / 2]$.

Remark 10.2. This corollary permits to guess how to lift $M$ to an operator that acts by convolution with a measure $\mu$. Note that $\mu$ is a Hahn-Banach extension of the linear form $f \mapsto M f(0)$. The relative multiplier $M$ is an isometry if and only if $t=0$ and $\mu$ is the Dirac measure in 0 . Otherwise, $t \neq 0$; the proof of Theorem $1.2(a)$ shows that $\mu$ is a linear combination $\alpha \delta_{y}+\beta \delta_{w}$ of two Dirac measures such that the norm of $M$ is $|\alpha|+|\beta|$. Let $f(x)=l \mathrm{e}^{-\mathrm{i} k x}+(k+$ $l) \mathrm{e}^{\mathrm{i} \pi /(k+l)}+k \mathrm{e}^{\mathrm{i} l x}: M$ attains its norm at $f, f$ attains its maximum modulus at 0 and $2 m \pi /(k+l)$, and $M f$ attains its maximum modulus at $2 m \pi /(k+l)$, where $m$ is the inverse of $l$ modulo $k+l$. As

$$
\begin{aligned}
(|\alpha|+|\beta|) \max _{x}|f(x)| & =\max _{x}|M f(x)| \\
& =|\mu * f(2 m \pi /(k+l))| \\
& =|\alpha f(2 m \pi /(k+l)-y)+\beta f(2 m \pi /(k+l)-w)|,
\end{aligned}
$$

we must choose $\{y, w\}=\{0,2 m \pi /(k+l)\}$. A computation yields then

$$
\mu=\mathrm{e}^{\mathrm{i} t / 2} \frac{\sin (\pi /(k+l)-t / 2)}{\sin (\pi /(k+l))} \delta_{0}+\mathrm{e}^{\mathrm{i}(t / 2+\pi /(k+l))} \frac{\sin (t / 2)}{\sin (\pi /(k+l))} \delta_{2 m \pi /(k+l)} .
$$

Consult [20] on this issue.

\section{The Sidon constant of integer sets}

Let us study the maximum modulus of a trigonometric trinomial with given Fourier coefficient moduli sum. We get the following result as an immediate consequence of Corollary 9.4 .

Proposition 11.1. Let $k$ and $l$ be two positive coprime integers. Let $r_{1}, r_{2}$ and $r_{3}$ be three positive real numbers. Let $t \in[0, \pi /(k+l)]$. Then

$$
\max _{x}\left|r_{1} \mathrm{e}^{-\mathrm{i} k x}+r_{2} \mathrm{e}^{\mathrm{i} t}+r_{3} \mathrm{e}^{\mathrm{i} l x}\right| \geqslant\left(r_{1}+r_{2}+r_{3}\right) \cos (\pi / 2(k+l))
$$

with equality if and only if $r_{1}: r_{2}: r_{3}=l: k+l: k$ and $t=\pi /(k+l)$.

This means that the Sidon constant of $\{-k, 0, l\}$ equals $\sec (\pi / 2(k+l))$.

The Sidon constant of integer sets was known in three instances only: 
- The equality

$$
\max _{x}\left|r_{1} \mathrm{e}^{\mathrm{i}\left(t_{1}+\lambda_{1} x\right)}+r_{2} \mathrm{e}^{\mathrm{i}\left(t_{2}+\lambda_{2} x\right)}\right|=r_{1}+r_{2}
$$

shows that the Sidon constant of sets with one or two elements is 1 .

- The Sidon constant of $\{-1,0,1\}$ is $\sqrt{2}$ and it is attained for $\mathrm{e}_{-1}+2 \mathrm{i}+\mathrm{e}_{1}$. Let us give the original argument: if $f(x)=\left|r_{1} \mathrm{e}^{-\mathrm{i} x}+r_{2} \mathrm{e}^{\mathrm{i} t}+r_{3} \mathrm{e}^{\mathrm{i} x}\right|^{2}$, the parallelogram identity and the arithmetic-quadratic inequality yield

$$
\begin{aligned}
\max _{x} f(x) & \geqslant \max _{x} \frac{f(x)+f(x+\pi)}{2} \\
& =\max _{x} \frac{\left|r_{1} \mathrm{e}^{-\mathrm{i} x}+r_{3} \mathrm{e}^{\mathrm{i} x}+r_{2} \mathrm{e}^{\mathrm{i} t}\right|^{2}+\left|r_{1} \mathrm{e}^{-\mathrm{i} x}+r_{3} \mathrm{e}^{\mathrm{i} x}-r_{2} \mathrm{e}^{\mathrm{i} t}\right|^{2}}{2} \\
& =\max _{x}\left|r_{1} \mathrm{e}^{-\mathrm{i} x}+r_{3} \mathrm{e}^{\mathrm{i} x}\right|^{2}+\left|r_{2} \mathrm{e}^{\mathrm{i} t}\right|^{2} \\
& =\left(r_{1}+r_{3}\right)^{2}+r_{2}^{2} \geqslant \frac{\left(r_{1}+r_{2}+r_{3}\right)^{2}}{2} .
\end{aligned}
$$

- The Sidon constant of $\{0,1,2,3,4\}$ is 2 and it is attained for $1+2 \mathrm{e}_{1}+$ $2 \mathrm{e}_{2}-2 \mathrm{e}_{3}+\mathrm{e}_{4}$.

These results were obtained by D. J. Newman (see [19].) The fact that the Sidon constant of integer sets with three elements cannot be 1 had been noted with pairwise different proofs in [19, 2, 9].

The following estimates for the Sidon constant of large integer sets are known.

- E. Beller and D. J. Newman [1] showed that the Sidon constant of $\{0,1$, $\ldots, n\}$ is equivalent to $\sqrt{n}$.

- (Hadamard sets.) Let $q>1$ and suppose that the sequence $\left(\lambda_{j}\right)_{j \geqslant 1}$ grows with geometric ratio $q:\left|\lambda_{j+1}\right| \geqslant q\left|\lambda_{j}\right|$ for every $j$. Then the Sidon constant of $\left\{\lambda_{1}, \lambda_{2}, \ldots\right\}$ is finite; it is at most 8 if $q \geqslant 2$, it is at most 2 if $q \geqslant 3$ (see [11]), and it is at most $1+\pi^{2} /\left(2 q^{2}-2-\pi^{2}\right)$ if $q>\sqrt{1+\pi^{2} / 2}$ (see [13, Corollary 9.4] or the updated [14, Corollary 10.2.1].)

Our computations show that the last estimate of the Sidon constant has the right order in $q^{-1}$ for geometric progressions.

Proposition 11.2. Let $C$ be the Sidon constant of the geometric progression $\left\{1, q, q^{2}, \ldots\right\}$, where $q \geqslant 3$ is an integer. Then

$$
1+\pi^{2} / 8(q+1)^{2} \leqslant \sec (\pi / 2(q+1)) \leqslant C \leqslant 1+\pi^{2} /\left(2 q^{2}-2-\pi^{2}\right) .
$$

One initial motivation for this work was to decide whether there are sets $\left\{\lambda_{j}\right\}_{j \geqslant 1}$ with $\left|\lambda_{j+1}\right| \geqslant q\left|\lambda_{j}\right|$ whose Sidon constant is arbitrarily close to 1 and to find evidence among sets with three elements. That there are such sets, 
arbitrarily large albeit finite, may in fact be proved by the method of Riesz products in [8, Appendix V, §1.II]; see also [14, Proposition 13.1.3]. The case of infinite sets remains open.

A second motivation was to show that the real and complex unconditional constants of the basis $\left(\mathrm{e}_{\lambda_{1}}, \mathrm{e}_{\lambda_{2}}, \mathrm{e}_{\lambda_{3}}\right)$ of $\mathscr{C}_{\Lambda}$ are different; we prove however that they coincide, and it remains an open question whether they may be different for larger sets. The real unconditional constant of $\left(\mathrm{e}_{\lambda_{1}}, \mathrm{e}_{\lambda_{2}}, \mathrm{e}_{\lambda_{3}}\right)$ is the maximum of the norm of the eight unimodular relative Fourier multipliers $\left(t_{1}, t_{2}, t_{3}\right)$ such that $t_{k}=0$ modulo $\pi$. Let $i, j, k$ be a permutation of $1,2,3$ such that the power of 2 in $\lambda_{i}-\lambda_{k}$ and in $\lambda_{j}-\lambda_{k}$ are equal. Lemma 2.1 shows that the four relative multipliers satisfying $t_{i}=t_{j}$ modulo $2 \pi$ are isometries and that the norm of any of the four others, satisfying $t_{i} \neq t_{j}$ modulo $2 \pi$, gives the real unconditional constant. In general, the complex unconditional constant is bounded by $\pi / 2$ times the real unconditional constant, as proved in [18; in our case, they are equal.

Corollary 11.3. The complex unconditional constant of the basis $\left(\mathrm{e}_{\lambda_{1}}, \mathrm{e}_{\lambda_{2}}\right.$, $\mathrm{e}_{\lambda_{3}}$ ) of $\mathscr{C}_{\Lambda}$ is equal to its real unconditional constant.

Acknowledgement. The Hungarian-French Scientific and Technological Governmental Cooperation, Project \#F-10/04, eased scientific contacts that were helpful to this work.

\section{References}

[1] E. Beller and D. J. Newman, An $l_{1}$ extremal problem for polynomials, Proc. Amer. Math. Soc. 29 (1971), 474-481.

[2] D. I. Cartwright, R. B. Howlett and J. R. McMullen, Extreme values for the Sidon constant, Proc. Amer. Math. Soc. 81 (1981), 531-537.

[3] N. G. Chebotarëv, On a general criterion of the minimax, C. R. (Doklady) Acad. Sci. URSS (N.S.) 39 (1943), 339-341.

[4] _ Kriteriǔ minimaksa i ego prilozheniya, in: Sobranie sochineniŭ. Vol. 2, Izdatel'stvo Akademii Nauk SSSR, Moscow-Leningrad, 1949, 396-409.

[5] V. F. Dem'yanov and V. N. Malozëmov, Introduction to minimax, Halsted Press [John Wiley \& Sons], New York-Toronto, 1974. Translated from the Russian by D. Louvish.

[6] K. M. Dyakonov, Extreme points in spaces of polynomials, Math. Res. Lett. 10 (2003), 717-728.

[7] S. Hartman, Some problems and remarks on relative multipliers, Colloq. Math. 54 (1987), 103-111. Corrected in Colloq. Math. 57 (1989), 189.

[8] J.-P. Kahane and R. Salem, Ensembles parfaits et séries trigonométriques, Actualités Sci. Indust. 1301, Hermann, Paris, 1963. 
[9] D. Li, Complex unconditional metric approximation property for $\mathscr{C}_{\Lambda}(\mathbb{T})$ spaces, Studia Math. 121 (1996), 231-247.

[10] J. E. Littlewood, A theorem on power series, Proc. London Math. Soc. (2) 23 (1925), 94-103.

[11] J. M. López and K. A. Ross, Sidon sets, Lecture Notes Pure Appl. Math. 13, Marcel Dekker Inc., New York, 1975.

[12] S. Neuwirth, The Sidon constant of sets with three elements. arxiv.org/ math/0102145, 2001.

[13] —, Metric unconditionality and Fourier analysis, Studia Math. 131 (1998), 19-62.

[14] - Multiplicateurs et analyse fonctionnelle, $\mathrm{PhD}$ thesis, Université Paris 6, 1999. tel.archives-ouvertes.fr/tel-00010399.

[15] G. Pólya and G. Szegö, Problems and theorems in analysis. Vol. I: Series, integral calculus, theory of functions, Grundlehren math. Wiss. 193, Springer-Verlag, New York, 1972. Translated from the German by D. Aeppli.

[16] Sz. Gy. Révész, Minimization of maxima of nonnegative and positive definite cosine polynomials with prescribed first coefficients, Acta Sci. Math. (Szeged) 60 (1995), 589-608.

[17] R. Salem, Sur les propriétés extrémales de certains polynomes trigonométriques, C. R. Acad. Sci. Paris 196 (1933), 1776-1778.

[18] J. A. Seigner, Rademacher variables in connection with complex scalars, Acta Math. Univ. Comenian. (N.S.) 66 (1997), 329-336.

[19] H. S. Shapiro, Extremal problems for polynomials and power series, Master's thesis, Massachusetts Institute of Technology, 1951.

[20] - On a class of extremal problems for polynomials in the unit circle, Portugal. Math. 20 (1961), 67-93.

[21] S. Straszewicz, Über exponierte Punkte abgeschlossener Punktmengen, Fund. Math. 24 (1935), 139-143.

Keywords. Trigonometric trinomial, maximum modulus, exposed point, extreme point, Mandel'shtam problem, extremal problem, relative Fourier multiplier, Sidon constant, unconditional constant.

2000 Mathematics Subject Classification. Primary 30C10, 42A05, 42A45, 46B20; Secondary 26D05, 42A55, 46B15.

Stefan Neuwirth, Laboratoire de Mathématiques, Université de Franche-Comté, 25030 Besançon cedex, France, stefan.neuwirth@univ-fcomte.fr. 